\title{
DETECÇÃO DO PADRÃO DE MOTRICIDADE FINA EM PACIENTES COM DOENÇA DE PARKINSON A PARTIR DE IMAGENS DIGITALIZADAS
}

\section{DETECTION OF STANDARD MOTRICITY THIN IN PATIENTS WITH PARKINSON'S DISEASE FROM SCANNED IMAGES}

Caio César Russi', Francisco Assis da Silva', Danillo Roberto Pereira', Mário Augusto Pazoti', Leandro Luiz de Almeida', Almir Olivette Artero $^{2}$

${ }^{1}$ Faculdade de Informática de Presidente Prudente - FIPP, Universidade do Oeste Paulista - UNOESTE. ${ }^{2}$ Faculdade de Ciências e Tecnologia - FCT, Universidade Estadual Paulista - Unesp

E-mail: almir@fct.unesp.br; caio.russi@hotmail.com, \{chico, danilopereira, mario, llalmeida\}@unoeste.br

RESUMO - O mal de Parkinson é uma doença neurodegenerativa que atua em uma região do cérebro responsável pela a coordenação dos movimentos, sendo uma das suas principais características o aparecimento de tremores nas mãos. Este trabalho apresenta uma metodologia para auxiliar no diagnóstico da doença de Parkinson a partir do traçado feito pelo paciente em uma espiral de Arquimedes. Na metodologia são utilizados alguns algoritmos de processamento de imagens na espiral desenhada pelo paciente, como o afinamento de Zhang-Suen responsável por esqueletizar o desenho da espiral e o algoritmo busca em largura para coletar os pontos da espiral. Foram extraídas cinco características no traçado do paciente com o intuito de classifica-lo com ou sem a doença de Parkinson a partir das técnicas de aprendizado de máquina KNN, Naive Bayes e SVM.

Palavras-chave: Parkinson; Espiral de Arquimedes; aprendizado de máquina.

ABSTRACT - Parkinson disease is a neurodegenerative disease which act in a region of the brain responsible for the coordination of movements, one of its main features is the appearance of hand tremors. This work presents a methodology to assist in the diagnosis of Parkinson disease from the tracing made by the patient in a spiral of Archimedes. In the methodology used are some image processing algorithms in a spiral drawn by the patient, as the Zhang-Suen thinning skeletonize responsible for drawing and the width spiral search algorithm to collect the points the spiral. Five characteristics were extracted from the patient's tracing with interest classify it with or

Recebido em: 20/04/2016 Revisado em: 10/10/2016 Aprovado em: 03/11/2016 without Parkinson disease from the machine learning techniques KNN, Naive Bayes and SVM.

Keywords: Parkinson; Spiral of Archimedes; machine learning. 


\section{INTRODUÇÃO}

A Doença de Parkinson (DP), também conhecida como Mal de Parkinson, é uma desordem neurológica degenerativa progressiva do sistema nervoso central. Uma de suas principais características é debilitar a capacidade do cérebro em controlar os movimentos corporais, sendo a mão um dos seus principais alvos segundo Bravo e Nassif (2014).

Segundo Forman, Trojanowski e Lee (2004), este é um dos principais e mais comuns distúrbios nervosos da terceira idade e em conjunto com outros problemas degenerativos, estão bem próximos a superar o câncer como a principal causa de morte.

O tremor causado pela doença nas mãos é descrito como tremor de repouso, que representa um tremor anormal (patológico), que pode ser interrompido no início de uma ação e que pode desaparecer durante o sono (MATTOS, 1998).

O desenho de uma espiral de Arquimedes é comumente utilizado na avaliação de pacientes com tremores patológicos e outras desordens do movimento, o que segundo Bain (1993) provou ser uma medida válida e conveniente da deficiência causada por tremores posturais (tremores de ação que aparecem ao escrever).

A espiral desenhada é então digitalizada no computador, o que permite aplicar técnicas de visão computacional e extrair características no traçado feito pelo paciente para serem utilizadas nos classificadores, e assim obter os resultados.

Este trabalho apresenta uma metodologia para o reconhecimento de padrões encontrados no traçado feito por pacientes na espiral de Arquimedes, e desta forma conseguir classificar um paciente com ou sem a doença. Para a classificar um paciente, foram utilizadas as técnicas de aprendizado de máquina KNN (K-Nearest Neighbor), Naive Bayes e o SVM (Support Vector Machine), de acordo com as características obtidas do traçado do pacientes: picos máximos, picos mínimos, angulação no traçado do paciente, RMS (Root Mean Square) e a soma das Distâncias Euclidianas.

O artigo está organizado da seguinte forma: a Seção 2 apresenta os trabalhos relacionados; na Seção 3 é apresentado o referencial teórico utilizado na metodologia; na Seção 4 é demonstrada a metodologia para o desenvolvimento do trabalho; na Seção 5 são discutidos os experimentos e os resultados; na Seção 6 são mostradas as conclusões do trabalho.

\section{TRABALHOS RELACIONADOS}

Nesta seção são apresentados os trabalhos relacionados ao desenvolvimento deste trabalho, que se referem à etapa de 
coleta dos pontos da espiral e das características extraídas.

No trabalho de Almeida (2011) é utilizada a conversão de coordenadas originais da espiral traçada pelo paciente para as coordenadas polares (raio, ângulo), em que é possível linearizar a espiral desenhada e compará-la com a espiral original, onde esta é expressada por uma reta. As características obtidas são a estimativa da atividade do tremor, onde primeiramente, os pontos são convertidos em coordenadas polares e então é calculada a diferença radial ponto a ponto da espiral traçada pelo paciente com a espiral original. A atividade do tremor utiliza o cálculo da estimativa da atividade do tremor para calcular o RMS (Root Mean Square) do sinal do tremor, que é a raiz quadrada da média dos tremores ao quadrado. Amplitude máxima pico-a-pico do sinal de tremor, esse valor representa a diferença entre o maior valor calculado na primeira característica com o menor valor da mesma característica, indicando que quanto maior a diferença, mais longe está a espiral traçada da original.

No trabalho de Pereira et al. (2015), a mocoleta dos pontos na espiral é realizado por meio da criação de 360 retas, cada uma com a diferença de um grau entre as suas adjacentes, tomando como base o ponto central da espiral e os ponto na espiral desenhada que forem colineares. Com essa técnica não é possível obter todos os pontos da espiral, devido de fato que quanto mais distante os pontos estão do centro da espiral, mais distantes estará uma reta das outras. As características extraídas foram o RMS (sendo a raiz quadrada da média da diferença radial), a diferença máxima entre todas as diferenças radiais, a diferença mínima entre todas as diferenças radiais, a Média do Tremor Relativa (MTR) quantificando a quantidade do tremor no traçado do paciente, o raio mínimo e o raio máximo. Os classificadores utilizados foram o SVM (Support Vector Machine), Naive Bayes e o OPF (Optimum-Path Forest).

\section{REFERENCIAL TEÓRICO}

Nesta seção é apresentado o referencial teórico utilizado neste trabalho.

\subsection{Espiral de Arquimedes}

A espiral de Arquimedes (Figura 1) é uma figura geométrica que apresenta uma distância de separação uniforme e igual a $2 \pi b$ em todas as suas voltas. Esta espécie de espiral pode ser representada pela seguinte fórmula em coordenadas polares, onde $r$ é o raio, $\theta$ o ângulo e $a$ e $b$ são constantes (ALMEIDA et al., 2010).

$$
r=a+b \theta
$$


De acordo com Almeida (2011), o traçado da espiral de Arquimedes é comumente utilizado para extrair características que possibilitam detectar os tremores cinéticos (tremor voluntário).

Figura 1 - Espiral de Arquimedes.

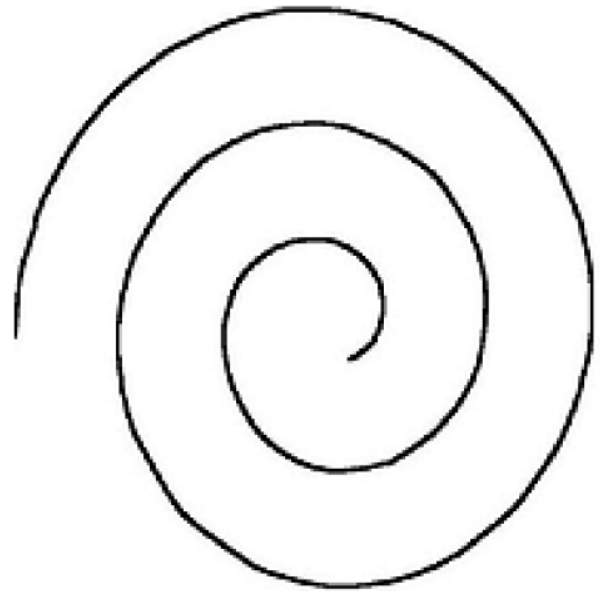

\subsection{Afinamento de Zhang-Suen}

Segundo Zhang e Suen (1984), a operação denominada afinamento remove todos os pixels redundantes em uma imagem, produzindo uma nova imagem simplificada com largura de um único pixel. O afinamento de Zhang-Suen funciona basicamente em binarizar uma imagem, onde os pontos de interesse da imagem ficarão com valor 1 e o restante com valor 0 .

A ideia básica do método Zhang-Suen é decidir se um determinado pixel será eliminado olhando somente seus oito vizinhos. Consiste em sucessivas aplicações de duas condições para decidir se o pixel deve ou não ser removido. Com isso os pixels do contorno da imagem são extraídos, exceto aqueles que pertencem ao objeto. Se as duas condições (Pseudocódigo

1) forem verdadeiras, então um pixel será removido da imagem.

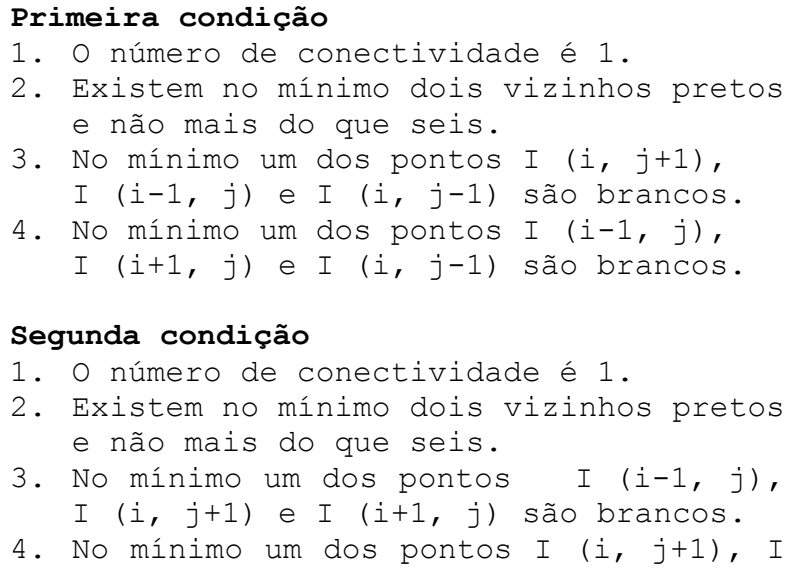

2. Existem no mínimo dois vizinhos pretos e não mais do que seis.

3. No mínimo um dos pontos $I(i-1, j)$, $I(i, j+1)$ e I $(i+1, j)$ são brancos.

4. No mínimo um dos pontos I (i, j+1), I $(i-1, j)$ e I $(i, j-1)$ são brancos.

Pseudocódigo 1. Duas condições de ZhangSuen.

Após as duas condições serem verdadeiras, o pixel é então removido e a conexidade do objeto não será comprometida (ZHANG; SUEN, 1984).

\subsection{Classificadores}

\subsubsection{SVM (Support Vector Machine)}

Proposto por Vapnik (1998), o SVM (Support Vector Machine) escolhe de um número infinito de classificadores lineares que separam os dados, o hiperplano que deixa a maior margem entre as duas classes. A margem é definida como a soma das distâncias do hiperplano a partir do ponto mais próximo das duas classes nomeados vetores de suporte. Tal hiperplano é chamado de Optimal Separating Hyperplane (OSH), (OSUNA; FREUND; GIROSI, 1997). O OSH tem a forma: 


$$
f(x)=\operatorname{sign}(w \cdot x+b),
$$

onde

$$
w=\sum_{i=1}^{l} \alpha_{i} y_{i} x_{i}
$$

e

$$
b=y_{j}-w \cdot x_{j}
$$

Por outro lado, se as classes não são linearmente separáveis, ainda é possível bloquear o hiperplano, que maximiza a margem e minimiza uma quantidade proporcional ao número de erros de classificação. Para estes casos generalizados da OSH, é determinado por meio da minimização:

$$
\Phi(w, £)=\frac{1}{2}\|w\|^{2}+C \sum_{i=1}^{l} £_{i},
$$

sujeito as seguintes restrições

$y_{i}\left[w \cdot x_{i}+b\right] \geq 1-€_{i,} £_{i} \geq 0, \quad i=1, \ldots, l$,

onde C é uma constante positiva que determina o trade-off entre margem e o erro de classificação.

\subsubsection{KNN (K-Nearest Neighbor)}

O KNN (K-Nearest Neighbor) é um dos algoritmos de classificação mais simples, onde cada elemento do conjunto de treinamento representa um ponto no espaço n-dimensional.

A classificação de um elemento é de acordo com a classe predominante dos Kvizinhos mais próximos. Foi utilizada a Distância Euclidiana para o cálculo da distância.
Sejam

$$
X=(x 1, x 2, \ldots, x n)
$$

e

$$
Y=(y 1, y 2, \ldots, y n),
$$

dois pontos do $R^{n}$, a Distância Euclidiana entre $\mathrm{X}$ e $\mathrm{Y}$ é dada por:

$d(X, Y)=\sqrt{\left(x_{1}-y_{1}\right)^{2}+\left(x_{2}-y_{2}\right)^{2}+\cdots+\left(x_{n}-y_{n}\right)^{n}}(9)$

\subsubsection{Naive Bayes}

Segundo Langley e Iba (1993), o classificador Naive Bayes faz a sua classificação por meio da probabilidade condicional. Ele verifica cada atributo das classes de forma isolada dos outros atributos, de tal foram que os atributos não tenham influência sobre os outros. O cálculo da probabilidade é dado pela seguinte fórmula:

$$
P[A \mid B]=\frac{P[B \mid A] * P[A]}{P[B]}
$$

A classificação de um elemento a uma determinada classe ocorre se a probabilidade dele pertencer a essa classe for maior do que a outra classe.

\section{METODOLOGIA}

Esta seção apresenta a metodologia desenvolvida neste trabalho, contendo o préprocessamento realizado na espiral traçada pelo paciente, coleta dos pontos da espiral e a extração das características. 


\subsection{Aquisição das Imagens}

As imagens utilizadas nesse trabalho,

(Figura 2), são de pacientes que foram diagnosticados com a doença e sem a doença. Os pacientes desenharam um modelo de espiral com caneta azul sobre o desenho de uma espiral impressa em preto.

Figura 2. a) Imagens de pacientes sem a doença de Parkinson. b) Imagens de pacientes com a doença de Parkinson.

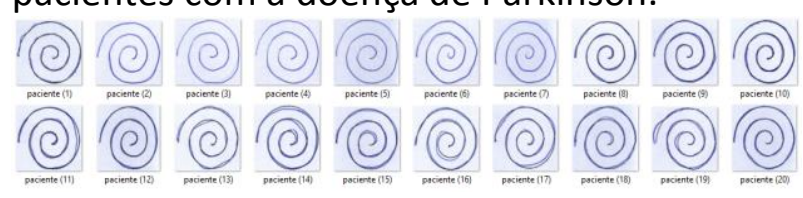

(a)

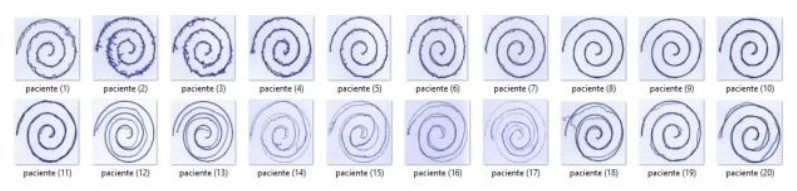

(b)

\subsection{Segmentação no traçado do paciente}

Este método consiste em separar o traçado da espiral desenhada pelo paciente da espiral original.

Um pixel não pertencente ao traçado do paciente é convertido para branco se os valores de $R, G$ e $B$ forem maiores que 200 , convertendo assim todos os pixels não pertencentes ao traçado do paciente, como a espiral original e o fundo da imagem, como mostra a Figura 3.
Figura 3. a) Imagem original da espiral desenhada pelo paciente. b) Espiral resultante da segmentação aplicada sobre a espiral a).

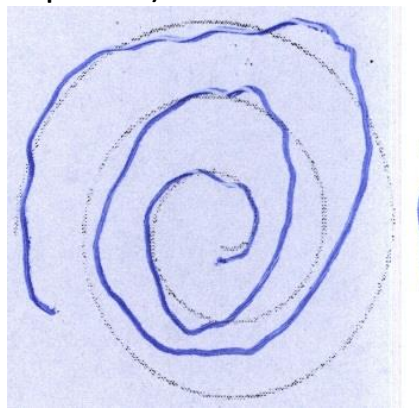

(a)

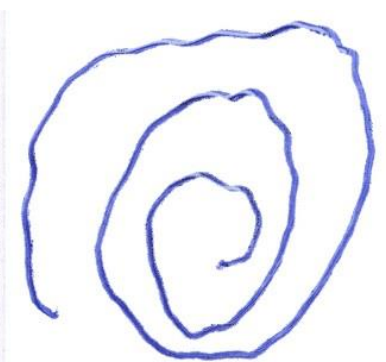

(b)

\subsection{Afinamento}

Esta etapa consiste em aplicar o afinamento de Zhang e Suen (1984) sobre o traçado do paciente, permitindo simplificar a largura do traçado feito por ele para um único pixel e com a garantia de que o esqueleto do objeto não seja alterado, permanecendo a conexidade da espiral. A imagem resultante é mostrada na Figura 4(b).

Figura 4. a) Traçado resultante da segmentação. b) Espiral esqueletizada resultante do algoritmo de Zhang-Suen aplicado sobre o traçado a).

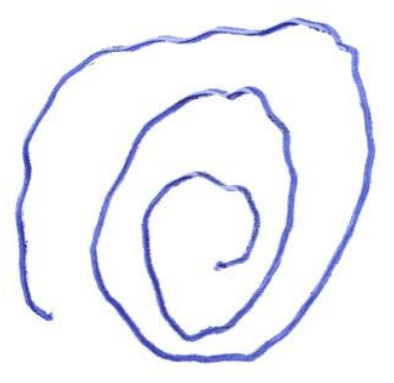

(a)

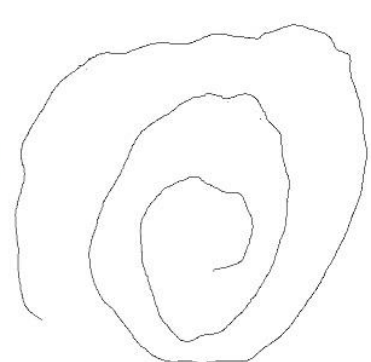

(b) 


\subsection{Busca em Largura}

O algoritmo de busca em largura foi utilizado para coletar os pontos na espiral desenhada pelo paciente após a segmentação e o afinamento, tendo como principal característica a sua expansão uniforme em largura, para obter todos os pontos da espiral de forma sequencial.

O início da busca utiliza o ponto central da espiral como o seu ponto de partida, coletando todos os pontos de forma sequencial até o último ponto localizado no outro extremo da espiral. O algoritmo é descrito no Pseudocódigo 2.

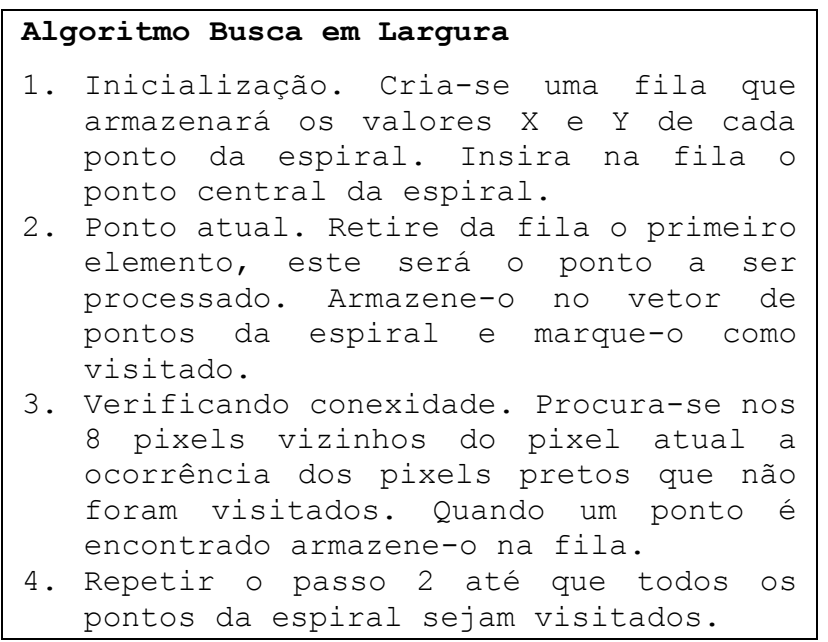

Pseudocódigo 2. Algoritmo de Busca em Largura.

\subsection{Equivalência entre os pontos}

O algoritmo de busca em largura coleta os pontos da espiral de forma sequencial, a partir do centro da espiral até a outra extremidade, mas não os garante que um ponto de índice $i$ da espiral desenhada seja equivalente ao ponto de mesmo índice no conjunto de pontos da espiral original.
Isto por que não se pode tomar como verdade que o traçado do paciente seja sempre em cima do traçado da espiral original.

Para encontrar quais pontos são equivalentes na espiral desenhada e na original, foi traçada uma reta sobre cada ponto da espiral original e o ponto central da mesma espiral, em seguida procura-se no conjunto de pontos coletados da espiral traçada pelo paciente os pontos colineares a estas retas formadas para encontrar quais são os pontos equivalentes na espiral traçada pelo paciente em relação a espiral original. Para o encontrar os pontos colineares foi utilizado o cálculo da determinante.

$$
\operatorname{Det}=\left|\begin{array}{lll}
X_{\text {OriCen }} & Y_{\text {OriCen }} & 1 \\
X_{\text {Ori }} & Y_{\text {Ori }} & 1 \\
X_{\text {Des }} & Y_{\text {Des }} & 1
\end{array}\right|
$$

A Figura 5 ilustra uma reta traçada em relação ao centro e um ponto da espiral original junto com os seus respectivos pontos relativos em ambas as espirais.

Figura 5. Exemplo de reta traçada e os respectivos pares de pontos em $A, B, C, D, E$ e F. 


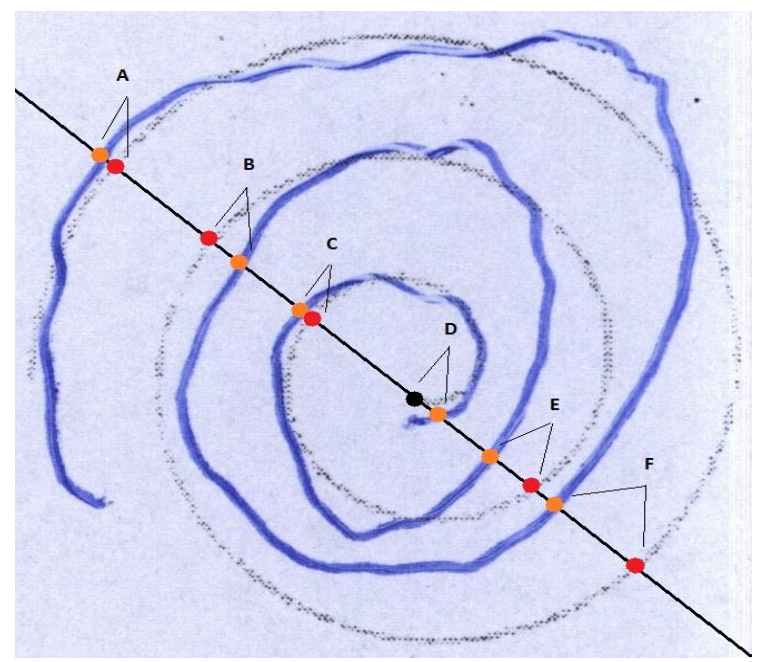

\subsection{Extração das características}

As características foram extraídas observando a diferença das imagens dos pacientes doentes e dos saudáveis. Ao total, foram extraídas cinco características, sendo elas: picos máximos e mínimos, angulação entre os vetores, RMS (Root Mean Square) e a soma das Distâncias Euclidianas, que se mostraram bem definidas de acordo com os resultados.

Para que a observação dessas características fosse possível, foi necessário linearizar as espirais desenhadas. A linearização no plano 2D é de acordo com a distância euclidiana de cada ponto da espiral do paciente com a espiral original. A espiral original foi linearizada paralela ao eixo $X$ e a espiral do paciente foi linearizada com uma proporção de distância equivalente ao desenho original. A Figura 6 ilustra uma espiral desenhada e a mesma linearizada.

\subsubsection{Picos máximos e mínimos}

Os picos máximos e mínimos são características definidas observando a variação e oscilação dos pontos das espirais quando linearizadas no plano 2D.

Um ponto é um pico máximo ou mínimo se o seu valor de $y$ for o maior/menor dentre todos os valores de $y$ dos pontos pertencentes a um determinado intervalo de pontos. A Figura 7 ilustra um pico máximo e mínimo.

Figura 6. a) Espiral desenhada por um paciente com a doença. b) Espiral linearizada resultante da espiral a).

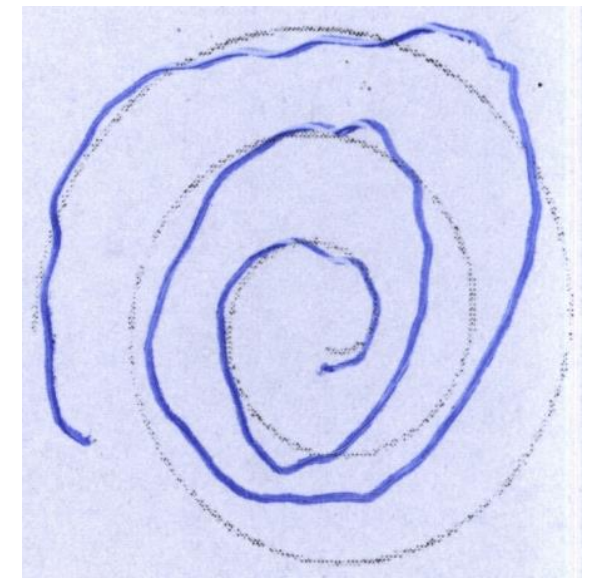

(a) 


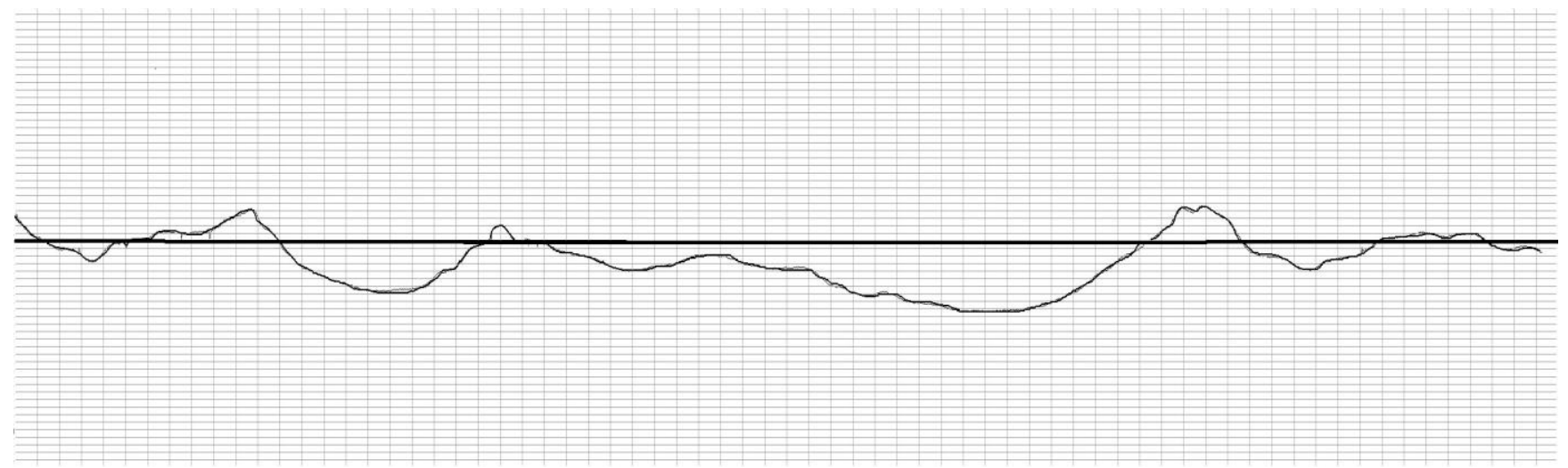

(b)

Figura 7. Exemplo de pico mínimo e máximo.

do ângulo entre os vetores é descrito a seguir:

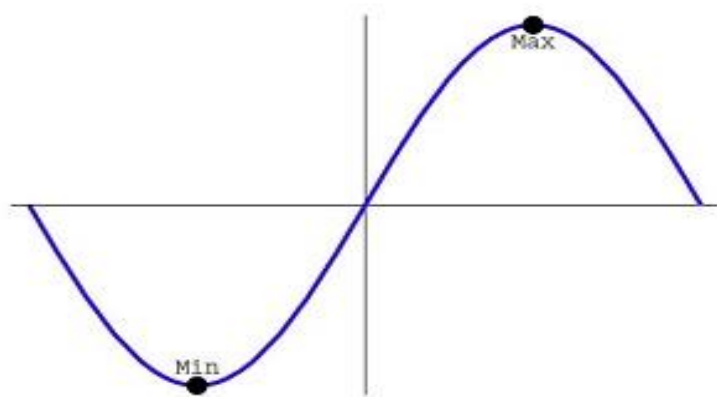

$$
\begin{aligned}
& \qquad \cos \theta=\frac{u . v}{|u| .|v|} \\
& \text { Foram contabilizados os ângulos }
\end{aligned}
$$

maiores que 12 graus para a característica.

\subsubsection{Angulação no traçado do Paciente}

A terceira característica também foi definida observando uma certa variação dos pontos da espiral linearizada. Tal variação corresponde ao quão brusca é a mudança de direção do traçado do paciente, mais especificamente o ângulo dessa mudança, se houver.

Para o cálculo do ângulo foram criados dois vetores, onde o primeiro vetor nomeado como $u$, foi criado a partir dos pontos $A$ e $C$ da espiral desenhada e o segundo vetor nomeado $v$, foi criado paralelo ao eixo $x$, com origem também em $A$, para auxiliar no cálculo do ângulo, como mostrado na Figura 8. A fórmula utilizada para o cálculo

Figura 8. Exemplo do ângulo $\theta$ entre os vetores $u$ e $v$, formados pelos pontos $(A, C)$ e $(A, B)$.

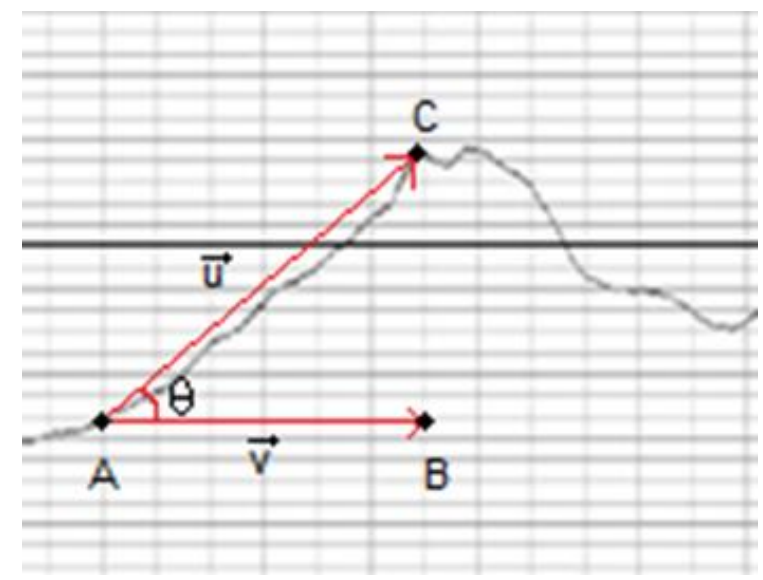

\subsubsection{RMS (Root Mean Square)}

A quarta característica foi calculada encontrando-se o valor de RMS (Root-Mean- 
Square), para os sinais de tremor nas coordenadas encontradas anteriormente.

$$
R M S=\sqrt{\frac{\sum_{i=1}^{N} d i s t_{i}^{2}}{N}}
$$

Segundo Almeida (2011), quanto maior este índice, mais distante estará a espiral desenhada da original. A fórmula utilizada no trabalho de Almeida (2011) foi modificada para este trabalho deixando de fazer uso das coordenadas polares, para ser utilizado o cálculo da Distância Euclidiana.

\subsubsection{Soma da Distância Euclidiana}

A quinta característica foi definida para obter uma margem significativa da classe dos pacientes com a doença dos pacientes sem a doença, visto que imagens de pacientes com a doença possuem um desvio maior em relação a espiral original. O cálculo desta característica é o somatório das Distâncias Euclidiana ponto a ponto.

$$
\text { Soma }=\sum_{k=1}^{N} \sqrt{\left(x_{\text {desk }}-x_{\text {orik }}\right)^{2}+\left(y_{\text {desk }}-y_{\text {orik }}\right)^{2}}
$$

\section{EXPERIMENTOS E RESULTADOS}

Foram utilizadas três técnicas de aprendizado de máquina para a realização dos experimentos, sendo eles o SVM (Support Vector Machine), KNN (K-Nearest Neighbors) e o Naive Bayes, descritos na secção 3.3. a

Foram utilizadas um total de 876 espirais desenhada por pacientes, sendo 696 de pacientes com a doença e 180 de pacientes sem a doença.

As imagens foram escolhidas com a porcentagem referente a cada classe e de forma aleatória para o treinamento.

A Tabela 1 apresenta os resultados de acertos e erros dos métodos KNN, Naive Bayes e SVM, com a quantidade de imagens utilizadas para treino e teste.

O SVM por se tratar de um método que tem o maior aproveitamento dentre os classificadores existentes, conseguiu obter a maior porcentagem de acerto. O KNN apesar de ser o mais simples dentre os três métodos, apresentou uma porcentagem de acerto bem próximo ao SVM. O Naive Bayes apesar de ter classificado bem os elementos, obteve o maior número de falsos positivos.

Aproximadamente $10 \%$ das imagens de pacientes com a doença em ambas as técnicas mostraram falsos negativos, o que indica que alguns pacientes não apresentaram tremor cinético ao realizar o traçado da espiral. E aproximadamente 7\% das imagens, com exceção do método Naive Bayes, apresentaram resultados de falsos positivos, isto pode levar em conta vários outros fatores envolvidos, como problemas de tremores não relacionado ao Parkinson, problemas de visão, coordenação motora, dentre outros. 
Tabela 1. Resumo dos experimentos para $10 \%$ a $90 \%$ das imagens para treinamento com a porcentagem de acerto e erro dos classificadores KNN, Naive Bayes e SVM.

\begin{tabular}{|c|c|c|c|c|c|c|c|c|c|}
\hline $\begin{array}{c}\text { Imagens } \\
\text { Treinamento }\end{array}$ & $\begin{array}{c}88 \\
(10 \%)\end{array}$ & $\begin{array}{c}176 \\
(20 \%)\end{array}$ & $\begin{array}{c}264 \\
(30 \%)\end{array}$ & $\begin{array}{c}352 \\
(40 \%)\end{array}$ & $\begin{array}{c}438 \\
(50 \%)\end{array}$ & $\begin{array}{c}526 \\
(60 \%)\end{array}$ & $\begin{array}{c}614 \\
(70 \%)\end{array}$ & $\begin{array}{c}701 \\
(80 \%)\end{array}$ & $\begin{array}{c}789 \\
(90 \%)\end{array}$ \\
\hline $\begin{array}{c}\text { Imagens } \\
\text { Teste }\end{array}$ & $\begin{array}{c}788 \\
(90 \%)\end{array}$ & $\begin{array}{c}700 \\
(80 \%)\end{array}$ & $\begin{array}{c}612 \\
(70 \%)\end{array}$ & $\begin{array}{c}524 \\
(60 \%)\end{array}$ & $\begin{array}{c}438 \\
(50 \%)\end{array}$ & $\begin{array}{c}350 \\
(40 \%)\end{array}$ & $\begin{array}{c}262 \\
(30 \%)\end{array}$ & $\begin{array}{c}175 \\
(20 \%)\end{array}$ & $\begin{array}{c}87 \\
(10 \%)\end{array}$ \\
\hline Acertos & & & & & & & & & \\
\hline KNN & 700 & 626 & 548 & 484 & 398 & 321 & 239 & 161 & 81 \\
& $(88,71 \%)$ & $(89,33 \%)$ & $(89,40 \%)$ & $(92,25 \%)$ & $(91,09 \%)$ & $(91,68 \%)$ & $(91.15 \%)$ & $(92,25 \%)$ & $(92,37 \%)$ \\
\hline Naive Bayes & 649 & 593 & 498 & 434 & 356 & 302 & 223 & 146 & 76 \\
& $(82,28 \%)$ & $(84,63 \%)$ & $(81,23 \%)$ & $(82,74 \%)$ & $(81,47 \%)$ & $(86,24 \%)$ & $(85,05 \%)$ & $(82,98 \%)$ & $(87,24 \%)$ \\
\hline SVM & 702 & 626 & 548 & 483 & 398 & 323 & 238 & 161 & 81 \\
& $(89,03 \%)$ & $(89,33 \%)$ & $(89,45 \%)$ & $(92,00 \%)$ & $(91,09 \%)$ & $(92,13 \%)$ & $(90,54 \%)$ & $(91,65 \%)$ & $(92,51 \%)$ \\
\hline Erros & & & & & & & & & 14 \\
\hline KNN & 88 & 74 & 64 & 40 & 38 & 29 & 23 & 6 \\
& $(11,29 \%)$ & $(10,67 \%)$ & $(10,60 \%)$ & $(7,75 \%)$ & $(8,91 \%)$ & $(8,32 \%)$ & $(8,85 \%)$ & $(7,75 \%)$ & $(7,63 \%)$ \\
\hline Naive Bayes & 149 & 107 & 114 & 90 & 80 & 48 & 39 & 29 & 11 \\
& $(17,72 \%)$ & $(15,37 \%)$ & $(18,77 \%)$ & $(17,26 \%)$ & $(18,53 \%)$ & $(13,76 \%)$ & $(14,95 \%)$ & $(17,02 \%)$ & $(12,76 \%)$ \\
\hline SVM & 86 & 74 & 64 & 41 & 38 & 27 & 24 & 14 & 6 \\
& $(10,97 \%)$ & $(10,67 \%)$ & $(10,55 \%)$ & $(8,00 \%)$ & $(8,91 \%)$ & $(7,87 \%)$ & $(9,45 \%)$ & $(8,35 \%)$ & $(7,49 \%)$ \\
\hline
\end{tabular}

\section{CONSIDERAÇÕES FINAIS}

Os métodos utilizados contribuíram de forma positiva para o resultado final. A segmentação foi fundamental para eliminar todos os pontos que não pertenciam ao traçado do paciente, eliminando qualquer tipo de problema com pontos não pertencentes a espiral desenhada. 0 afinamento de Zhang-Suen foi importante para a aplicação da busca em largura, justamente por deixar a espiral com a largura de 1 único pixel e com apenas um caminho do centro da espiral ao outro ponto extremo da espiral e a busca em largura por coletar todos os pontos da espiral, conseguindo assim as características com mais precisão. 0 método equivalência entre os pontos permitiu ser encontrado o ponto equivalente ao ponto original, podendo assim ter 0 cálculo da distância entre um ponto da desenhada e um ponto da original.

Os resultados foram satisfatórios nos classificadores KNN, Naive Bayes e SVM, com ressalto para o classificador SVM que obteve a maior porcentagem de acerto, por se tratar de um classificador mais robusto.

Futuros trabalhos podem ser desenvolvidos aplicando novas técnicas de extração das características, bem como fórmulas já existentes para a quantificação do tremor na espiral desenhada, para assim conseguir separar cada vez mais uma classe da outra.

\section{REFERÊNCIAS}

ALMEIDA, M. F. S. Análise temporal da relação entre o tremor fisiológico cinético e o envelhecimento com base em desenhos digitalizados da Espiral de Arquimedes. Universidade Federal de Uberlândia, 2011. 
ALMEIDA, M. F. S.; CAVALHEIRO, G. L.; PAIVA, L. R. M.; ANDRADE, A. O.; PEREIRA, A. A. Estudo do Tremor Cinético Através da Análise De Desenhos Manuais. VIII CEEL Conferência de Estudos em Engenharia Elétrica, Uberlândia - Minas Grais, 2010.

BAIN, P. G. et al. Assessing tremor severity. J Neurol Neurosurg Psychiatry, 1993.

BRAVO, P. A. F.; NASSIF, N.C., Doença de Parkinson: Terapêutica atual e avançada. 2014. Laboratório de Neurofarmacologia Molecular, Universidad de Chile. Disponível em:

<http://www.cff.org.br/sistemas/geral/revist a/pdf/12/inf25a29.pdf>. Acesso em: 2 ago. 2015.

FORMAN, M. S.; TROJANOWSKI, J. Q.; LEE, V. $M$. Neurodegenerative diseases: a decade of discoveries paves the way for therapeutic breakthroughs. Nat. Med., v. 10, 2004.

LANGLEY, P.; IBA, W.; THOMPSON, K. An analysis of bayesian classiers. In: THE TENTH NATIONAL CONFERENCE ON ARTICIAL INTEL LIGENCE. Proceedings... AAAI Press and MIT Press, 1993, p. 223-228.

MATTOS, J. P. Diagnóstico diferencial dos tremores. Arq. Neuro-Psiquiatr, v.56, no.2, p.320-323, jun. 1998. Disponível em: <http://www.scielo.br/scielo.php?pid=s0004282x1998000200027\&script=sci_arttext $>$.

Acesso em: 2 ago. 2015.

OSUNA, E.; FREUND, R.; GIROSI, F. Training support vector machines: an application to face detection. In: COMPUTER VISION AND PATTERN RECOGNITION, 1997. Proceedings... IEEE Computer Society Conference on, Jun 1997. p. 130-136.

PEREIRA, D. R. et al. A step Towards the Automated Diagnosis of Parkinson's Disease: Analyzing Handwriting Movements. In: COMPUTER-BASED MEDICAL SYSTEMS
(CBMS), IEEE INTERNATIONAL SYMPOSIUM ON, 28. Proceedings... 2015, p. 171-17.

VAPNIK, V. N. Statistical Learning Theory. New York: Wiley-Interscience, 1998.

ZHANG, T. Y.; SUEN, C. Y. A fast parallel algorithm for thinning digital patterns. Communications of the ACM, v. 27, p. 236239, 1984. 